Acta Crystallographica Section E

Structure Reports

Online

ISSN 1600-5368

\section{William T. A. Harrison}

Department of Chemistry, University of

Aberdeen, Meston Walk, Aberdeen AB24 3UE,

Scotland

Correspondence e-mail:

w.harrison@abdn.ac.uk

\section{Key indicators}

Single-crystal X-ray study

$T=293 \mathrm{~K}$

Mean $\sigma(\mathrm{C}-\mathrm{C})=0.003 \AA$

$R$ factor $=0.042$

$w R$ factor $=0.131$

Data-to-parameter ratio $=24.7$

For details of how these key indicators were automatically derived from the article, see

http://journals.iucr.org/e.

\title{
Piperizinium hydrogen phosphite monohydrate
}

The title compound, $\mathrm{C}_{6} \mathrm{H}_{12} \mathrm{~N}_{2}{ }^{2+} \cdot \mathrm{HPO}_{3}{ }^{2-} \cdot \mathrm{H}_{2} \mathrm{O}$, contains doubly protonated piperizinium cations, hydrogen phosphite anions and water molecules. The component species have normal geometrical parameters and interact by way of $\mathrm{N}-\mathrm{H} \cdots \mathrm{O}$ and $\mathrm{O}-\mathrm{H} \cdots \mathrm{O}$ hydrogen bonds, resulting in [010] chains of alternating $\left[\mathrm{HPO}_{3}\right]^{2-}$ and $\mathrm{H}_{2} \mathrm{O}$ species, crosslinked by the organic moieties. A possible $\mathrm{C}-\mathrm{H} \cdots \mathrm{O}$ interaction is also present.

\section{Comment}

The crystal structures of (protonated) amine hydrogen phosphites containing $\left[\mathrm{HPO}_{3}\right]^{2-}$ or $\left[\mathrm{H}_{2} \mathrm{PO}_{3}\right]^{-}$oxo-anions are of crystallochemical interest in terms of the interplay between the hydrogen bonds linking the cations, anions, and, if applicable, water molecules together (Averbuch-Pouchot, 1993a,b; Harrison, 2003a,b).

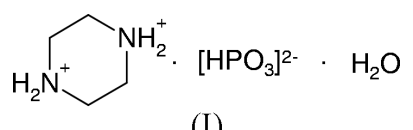

(I)

The asymmetric unit of the title compound, (I), consists of two half-molecule $\left\{\mathrm{C}_{2} \mathrm{H}_{6} \mathrm{~N}\right\}$ fragments of $\left(\mathrm{C}_{4} \mathrm{H}_{12} \mathrm{~N}_{2}\right)^{2+}$ piperizinium cations, an $\left[\mathrm{HPO}_{3}\right]^{2-}$ hydrogen phosphite group and a water molecule. Inversion symmetry (Fig. 1) generates the two complete piperizinium cations, and the water $\mathrm{O}$ atom is disordered over two adjacent sites (see Experimental). The

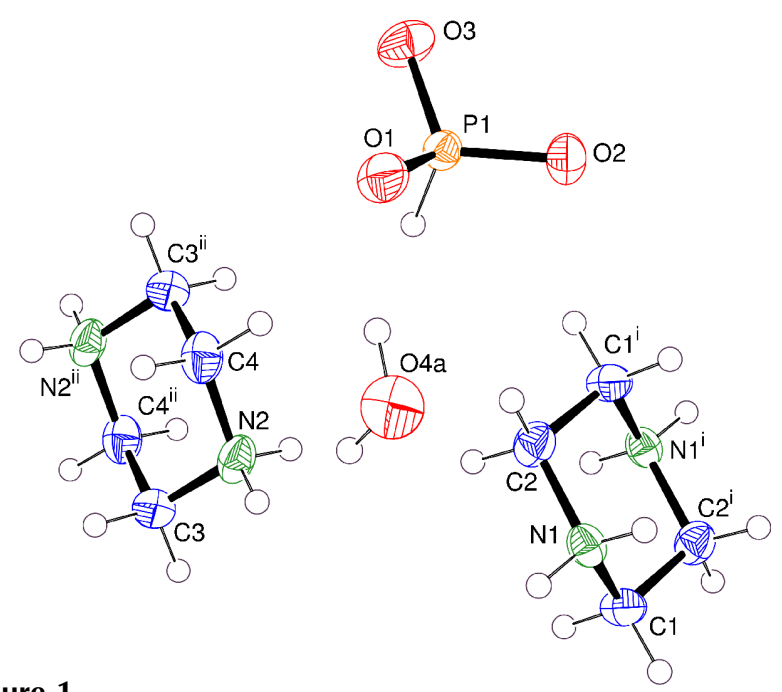

Figure 1

View of (I) (50\% displacement ellipsoids; $\mathrm{H}$ atoms are drawn as small spheres of arbitrary radius). The disordered $\mathrm{O} 4 b$ species is omitted. Symmetry codes: (i) $-x, 1-y,-z$; (ii) $1-x, 1-y,-z$.
Received 9 August 2004 Accepted 13 August 2004 Online 21 August 2004 


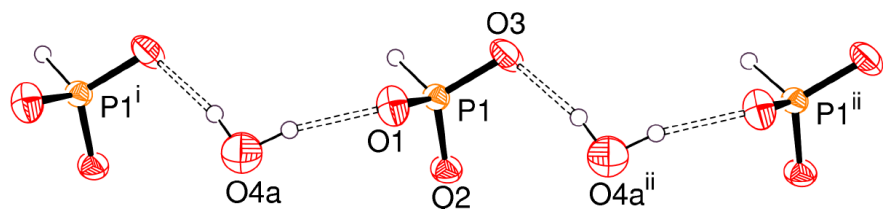

Figure 2

Detail of a [010] hydrogen phosphite-water chain with the $\mathrm{H} \cdots \mathrm{O}$ components of the hydrogen bonds indicated by dashed lines (atom $\mathrm{O} 4 b$ not shown). Symmetry codes: (i) $x, y+1, z$; (ii) $x, y-1, z$.

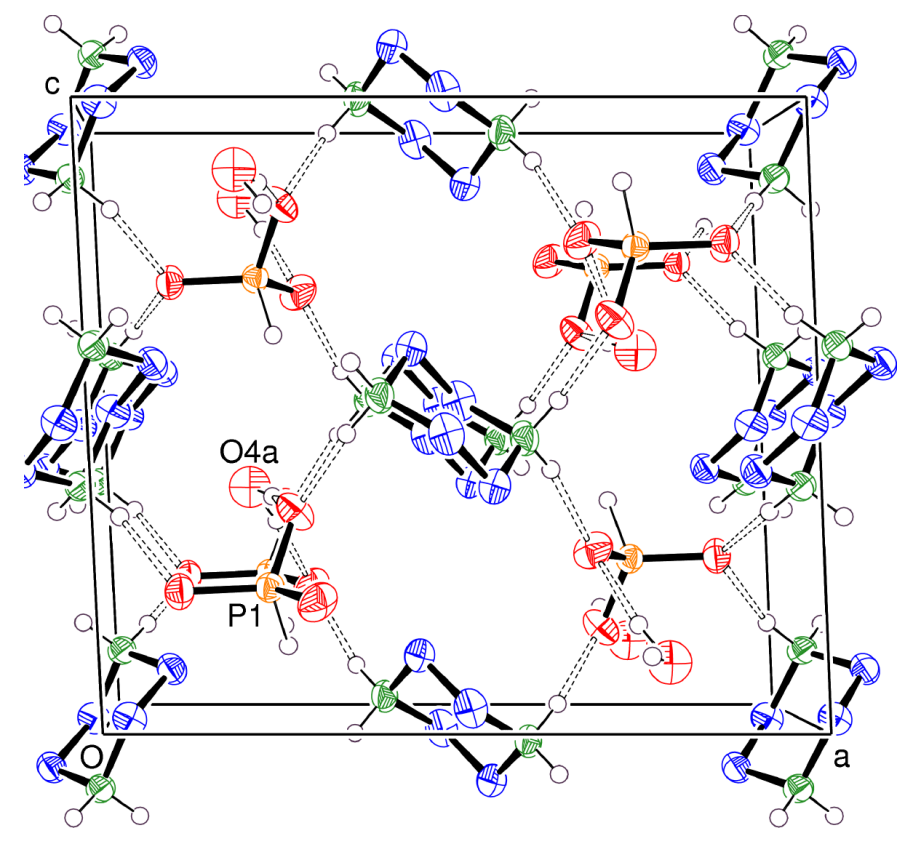

Figure 3

Unit-cell packing in (I) projected onto (010). The $\mathrm{H} \cdots \mathrm{O}$ components of the hydrogen bonds are indicated by dashed lines. $\mathrm{O} 4 b$ and all $\mathrm{C}-\mathrm{H} \mathrm{H}$ atoms are omitted for clarity.

hydrogen phosphite group shows its usual (Harrison, 2003a) pseudo-pyramidal geometry [mean $d(\mathrm{P}-\mathrm{O})=1.521(2) \AA$; mean $\left.\theta(\mathrm{O}-\mathrm{P}-\mathrm{O})=112.48(9)^{\circ}\right]$ and the organic species adopt typical chair conformations.

As well as electrostatic forces, the component species in (I) interact by means of $\mathrm{O}-\mathrm{H} \cdots \mathrm{O}$ and $\mathrm{N}-\mathrm{H} \cdots \mathrm{O}$ hydrogen bonds (Table 2), and possibly a $\mathrm{C}-\mathrm{H} \cdots \mathrm{O}$ interaction (see below). Infinite chains of alternating $\left[\mathrm{HPO}_{3}\right]^{2-}$ and $\mathrm{H}_{2} \mathrm{O}$ moieties are formed (Fig. 2) along [010] as a result of the water-to-phosphite $\mathrm{O}-\mathrm{H} \cdots \mathrm{O}$ hydrogen bonds, with the repeating units generated by translation symmetry. The resulting P1...P1 $1^{\text {ii }}$ (Fig. 2; see Table 2 for symmetry code) separation of 6.5706 (7) $\AA$ is naturally much larger than the typical P...P separations (4.7-4.9 $\mathrm{A}$ ) seen when $\left[\mathrm{H}_{2} \mathrm{PO}_{3}\right]^{-}$ dihydrogen phosphite units link together by way of $\mathrm{P}-\mathrm{O}-$ $\mathrm{H} \cdots \mathrm{O}-\mathrm{P}$ interactions without an intervening water molecule (Averbuch-Pouchot, 1993a, Harrison, 2003a).

The piperizinium cations crosslink the $[010]\left[\mathrm{HPO}_{3}\right]^{2-}-\mathrm{H}_{2} \mathrm{O}$ chains by way of the $\mathrm{N}-\mathrm{H} \cdots \mathrm{O}$ hydrogen bonds (Table 2), with all four bonds close to linear [mean $\theta(\mathrm{N}-\mathrm{H} \cdots \mathrm{O})=168^{\circ}$ ]. A short $\mathrm{C} 1-\mathrm{H} 5 \cdots \mathrm{O} 4 a^{\mathrm{iv}}$ (Table 2$)$ interaction was identified in a PLATON (Spek, 2003) analysis of (I). If it is not merely a packing artefact, it may provide some additional coherence between the piperizinium cations and the water component of the $\left[\mathrm{HPO}_{3}\right]^{2-}-\mathrm{H}_{2} \mathrm{O}[010]$ chains, although its role, if any, in the disordering of the water molecule $\mathrm{O} 4$ atom is not obvious.

\section{Experimental}

$\mathrm{H}_{3} \mathrm{PO}_{3} \quad(0.82 \mathrm{~g} ; 1 \mathrm{mmol})$ and piperizine hexahydrate $(1.92 \mathrm{~g}$; $0.01 \mathrm{mmol}$ ) were dissolved in $10 \mathrm{ml}$ deionized water, resulting in a clear solution. Block-shaped crystals of (I) grew as the water evaporated over several days.

Crystal data

$\mathrm{C}_{6} \mathrm{H}_{12} \mathrm{~N}_{2}{ }^{2+} \cdot \mathrm{HPO}_{3}{ }^{2-} \cdot \mathrm{H}_{2} \mathrm{O}$

$M_{r}=186.15$

Monoclinic, $P 2_{1} / c$

$a=12.2476(8) \AA$

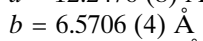

$c=10.6592(8) \AA$

$\beta=92.744(1)^{\circ}$

$V=856.8(1) \AA^{3}$

$Z=4$

$D_{x}=1.443 \mathrm{Mg} \mathrm{m}^{-3}$

Mo $K \alpha$ radiation

Cell parameters from 2470

reflections

$\theta=3.3-29.8^{\circ}$

$\mu=0.30 \mathrm{~mm}^{-1}$

$T=293(2) \mathrm{K}$

Block, colourless

$0.27 \times 0.23 \times 0.19 \mathrm{~mm}$

\section{Data collection}

Bruker SMART1000 CCD

diffractometer

$\omega$ scans

Absorption correction: multi-scan

(SADABS; Bruker, 1999)

$T_{\min }=0.925, T_{\max }=0.949$

6211 measured reflections

2468 independent reflections

1930 reflections with $I>2 \sigma(I)$

$R_{\text {int }}=0.022$

$\theta_{\text {max }}=30.0^{\circ}$

$h=-17 \rightarrow 16$

$k=-8 \rightarrow 9$

$l=-14 \rightarrow 12$

\section{Refinement}

Refinement on $F^{2}$

$R\left[F^{2}>2 \sigma\left(F^{2}\right)\right]=0.042$

$w R\left(F^{2}\right)=0.131$

$S=1.02$

2468 reflections

100 parameters

$\mathrm{H}$-atom parameters constrained $w=1 /\left[\sigma^{2}\left(F_{o}^{2}\right)+(0.0845 P)^{2}\right]$

where $P=\left(F_{o}{ }^{2}+2 F_{c}{ }^{2}\right) / 3$

$(\Delta / \sigma)_{\max }<0.001$

$\Delta \rho_{\max }=0.78 \mathrm{e}^{-3}$

$\Delta \rho_{\min }=-0.44 \mathrm{e}^{-3}$

Table 1

Selected bond lengths ( $\mathrm{A})$ for (I).

\begin{tabular}{llll}
\hline $\mathrm{P} 1-\mathrm{O} 3$ & $1.5151(13)$ & $\mathrm{P} 1-\mathrm{O} 1$ & $1.5234(14)$ \\
$\mathrm{P} 1-\mathrm{O} 2$ & $1.5230(12)$ & & \\
\hline
\end{tabular}

Table 2

Hydrogen-bonding geometry $\left(\AA,^{\circ}\right)$ for (I).

\begin{tabular}{lllll}
\hline$D-\mathrm{H} \cdots A$ & $D-\mathrm{H}$ & $\mathrm{H} \cdots A$ & $D \cdots A$ & $D-\mathrm{H} \cdots A$ \\
\hline $\mathrm{N} 1-\mathrm{H} 2 \cdots \mathrm{O} 22^{\mathrm{i}}$ & 0.90 & 1.84 & $2.7147(19)$ & 164 \\
$\mathrm{~N} 1-\mathrm{H} 3 \cdots \mathrm{O} 2^{\text {ii }}$ & 0.90 & 1.81 & $2.7043(19)$ & 172 \\
$\mathrm{~N} 2-\mathrm{H} 8 \cdots 3^{\text {ii }}$ & 0.90 & 1.77 & $2.642(2)$ & 163 \\
$\mathrm{~N} 2-\mathrm{H} 9 \cdots \mathrm{O} 1^{\mathrm{iii}}$ & 0.90 & 1.78 & $2.676(2)$ & 171 \\
$\mathrm{O} 4 a-\mathrm{H} 14 \cdots \mathrm{O} 1$ & 0.95 & 1.90 & $2.840(4)$ & 167 \\
$\mathrm{O} 4 a-\mathrm{H} 15 \cdots \mathrm{O} 3{ }^{\text {ii }}$ & 0.93 & 1.90 & $2.811(4)$ & 168 \\
$\mathrm{O} 4 b-\mathrm{H} 14 \cdots \mathrm{O} 1$ & 0.93 & 1.90 & $2.752(4)$ & 151 \\
$\mathrm{O} 4 b-\mathrm{H} 15 \cdots \mathrm{O} 3^{\text {ii }}$ & 0.96 & 1.90 & $2.765(4)$ & 149 \\
$\mathrm{C} 1-\mathrm{H} 5 \cdots \mathrm{O} 4 a^{\text {iv }}$ & 0.97 & 2.38 & $3.300(5)$ & 159 \\
\hline
\end{tabular}

Symmetry codes: (i) $-x, \frac{1}{2}+y, \frac{1}{2}-z$; (ii) $x, 1+y, z$; (iii) $x, \frac{1}{2}-y, z-\frac{1}{2}$; (iv) $-x, \frac{1}{2}+y, \frac{1}{2}-z$.

The water $\mathrm{O}$ atom was modelled as being disordered over two adjacent sites with isotropic displacement factors $[d(\mathrm{O} 4 a \cdots \mathrm{O} 4 b)=$ 0.638 (5) $\AA$; fractional site occupancies $=0.563$ (14) and 0.437 (14) for $\mathrm{O} 4 \mathrm{a}$ and $\mathrm{O} 4 b$, respectively, with their sum constrained to unity]. The present data did not reveal $\mathrm{H}$-atom sites that could be unambiguously associated with either $\mathrm{O} 4 \mathrm{a}$ or $\mathrm{O} 4 b$; instead, two distinct features in 


\section{organic papers}

the difference map provided $\mathrm{H}$-atom sites that were reasonable for both $\mathrm{O} 4 a$ and $\mathrm{O} 4 b$ (see Table 2). These $\mathrm{O}-\mathrm{H} \mathrm{H}$ atoms were refined by riding on $\mathrm{O} 4 a$ in their as-found positions. The $\mathrm{N}-\mathrm{H} \mathrm{H}$ atoms were found in difference maps and refined by riding in their idealized positions $[d(\mathrm{~N}-\mathrm{H})=0.90 \AA]$. The $\mathrm{H}$ atoms bonded to $\mathrm{C}$ and $\mathrm{P}$ were placed in calculated positions $[d(\mathrm{C}-\mathrm{H})=0.97 \AA ; d(\mathrm{P}-\mathrm{H})=1.32 \AA]$ and refined by riding. For all $\mathrm{H}$ atoms, the constraint $U_{\text {iso }}(\mathrm{H})=$ $1.2 U_{\text {eq }}$ (carrier atom) was applied.

Data collection: SMART (Bruker, 1999); cell refinement: SAINT (Bruker, 1999); data reduction: $S A I N T$; program(s) used to solve structure: SHELXS97 (Sheldrick, 1997); program(s) used to refine structure: SHELXL97; molecular graphics: ORTEP-3 (Farrugia,
1997); software used to prepare material for publication: SHELXL97.

\section{References}

Averbuch-Pouchot, M. T. (1993a) Acta Cryst. C49, 813-815.

Averbuch-Pouchot, M. T. (1993b) Acta Cryst. C49, 815-818.

Bruker (1999). SMART (Version 5.624), SAINT-Plus (Version 6.02A) and $S A D A B S$. Bruker AXS Inc., Madison, Wisconsin, USA.

Farrugia, L. J. (1997). J. Appl. Cryst. 30, 565-565.

Harrison, W. T. A. (2003a) Acta Cryst. E59, o769-o770.

Harrison, W. T. A. (2003b) Acta Cryst. E59, o1267-o1269.

Sheldrick, G. M. (1997). SHELXS97 and SHELXL97. University of Göttingen, Germany.

Spek, A. L. (2003). J. Appl. Cryst. 36, 7-13. 


\title{
supporting information
}

Acta Cryst. (2004). E60, o1577-o1579 [https://doi.org/10.1107/S1600536804020185]

\section{Piperizinium hydrogen phosphite monohydrate}

\author{
William T. A. Harrison
}

(I)

\section{Crystal data}

$\left(\mathrm{C}_{6} \mathrm{H}_{12} \mathrm{~N}_{2}\right)^{2+} \cdot\left[\mathrm{HPO}_{3}\right]^{2-} \cdot \mathrm{H}_{2} \mathrm{O}$

$M_{r}=186.15$

Monoclinic, $P 2_{1} / c$

Hall symbol: $-\mathrm{P} 2 \mathrm{ybc}$

$a=12.2476(8) \AA$

$b=6.5706(4) \AA$

$c=10.6592(8) \AA$

$\beta=92.744(1)^{\circ}$

$V=856.8(1) \AA^{3}$

$Z=4$

$$
F(000)=400
$$

$D_{\mathrm{x}}=1.443 \mathrm{Mg} \mathrm{m}^{-3}$
Mo $K \alpha$ radiation, $\lambda=0.71073 \AA$

Cell parameters from 2470 reflections

$\theta=3.3-29.8^{\circ}$

$\mu=0.30 \mathrm{~mm}^{-1}$

$T=293 \mathrm{~K}$

Block, colourless

$0.27 \times 0.23 \times 0.19 \mathrm{~mm}$

\section{Data collection}

Bruker SMART1000 CCD diffractometer

Radiation source: normal-focus sealed tube

6211 measured reflections

2468 independent reflections

Graphite monochromator

$\omega$ scans

Absorption correction: multi-scan

(SADABS; Bruker, 1999)

$T_{\min }=0.925, T_{\max }=0.949$

1930 reflections with $I>2 \sigma(I)$

$R_{\text {int }}=0.022$

$\theta_{\max }=30.0^{\circ}, \theta_{\min }=3.3^{\circ}$

$h=-17 \rightarrow 16$

$k=-8 \rightarrow 9$

$l=-14 \rightarrow 12$

\section{Refinement}

Refinement on $F^{2}$

Least-squares matrix: full

$R\left[F^{2}>2 \sigma\left(F^{2}\right)\right]=0.042$

$w R\left(F^{2}\right)=0.131$

$S=1.02$

2468 reflections

100 parameters

0 restraints

Primary atom site location: structure-invariant direct methods

Secondary atom site location: difference Fourier map

Hydrogen site location: difmap (O-H and N-H) and geom $(\mathrm{C}-\mathrm{H}$ and $\mathrm{P}-\mathrm{H})$

$\mathrm{H}$-atom parameters constrained

$w=1 /\left[\sigma^{2}\left(F_{\mathrm{o}}^{2}\right)+(0.0845 P)^{2}\right]$

where $P=\left(F_{0}^{2}+2 F_{\mathrm{c}}^{2}\right) / 3$

$(\Delta / \sigma)_{\max }<0.001$

$\Delta \rho_{\max }=0.78 \mathrm{e} \AA^{-3}$

$\Delta \rho_{\min }=-0.44$ e $\AA^{-3}$

Special details

Geometry. All e.s.d.'s (except the e.s.d. in the dihedral angle between two 1.s. planes) are estimated using the full covariance matrix. The cell e.s.d.'s are taken into account individually in the estimation of e.s.d.'s in distances, angles and torsion angles; correlations between e.s.d.'s in cell parameters are only used when they are defined by crystal symmetry. An approximate (isotropic) treatment of cell e.s.d.'s is used for estimating e.s.d.'s involving 1.s. planes. 
Refinement. Refinement of $F^{2}$ against ALL reflections. The weighted $R$-factor $w R$ and goodness of fit $S$ are based on $F^{2}$, conventional $R$-factors $R$ are based on $F$, with $F$ set to zero for negative $F^{2}$. The threshold expression of $F^{2}>\sigma\left(F^{2}\right)$ is used only for calculating $R$-factors(gt) etc. and is not relevant to the choice of reflections for refinement. $R$-factors based on $F^{2}$ are statistically about twice as large as those based on $F$, and $R$ - factors based on ALL data will be even larger.

Fractional atomic coordinates and isotropic or equivalent isotropic displacement parameters $\left(\AA^{2}\right)$

\begin{tabular}{llllll}
\hline & $x$ & $y$ & $z$ & $U_{\text {iso }} / U_{\text {eq }}$ & Occ. $(<1)$ \\
\hline P1 & $0.23916(3)$ & $-0.02316(6)$ & $0.23270(4)$ & $0.02544(15)$ & \\
H1 & 0.2609 & 0.0944 & 0.1366 & $0.031^{*}$ & \\
O1 & $0.27883(11)$ & $0.0919(2)$ & $0.35030(14)$ & $0.0398(3)$ & \\
O2 & $0.11571(10)$ & $-0.0529(2)$ & $0.22692(13)$ & $0.0335(3)$ & \\
O3 & $0.30154(11)$ & $-0.2194(2)$ & $0.21505(14)$ & $0.0420(4)$ & \\
N1 & $0.01729(11)$ & $0.6267(2)$ & $0.10922(13)$ & $0.0272(3)$ & \\
H2 & -0.0364 & 0.5840 & 0.1576 & $0.033^{*}$ & \\
H3 & 0.0549 & 0.7250 & 0.1512 & $0.033^{*}$ & \\
C1 & $-0.03148(15)$ & $0.7113(3)$ & $-0.01021(17)$ & $0.0318(4)$ & \\
H4 & 0.0258 & 0.7674 & -0.0596 & $0.038^{*}$ & \\
H5 & -0.0817 & 0.8203 & 0.0082 & $0.038^{*}$ & \\
C2 & $0.09189(15)$ & $0.4532(3)$ & $0.08474(18)$ & $0.0321(4)$ & \\
H6 & 0.1205 & 0.3975 & 0.1639 & $0.039^{*}$ & \\
H7 & 0.1530 & 0.5012 & 0.0383 & $0.039^{*}$ & \\
N2 & $0.39969(11)$ & $0.5910(2)$ & $0.03315(15)$ & $0.0339(4)$ & \\
H8 & 0.3613 & 0.6719 & 0.0831 & $0.041^{*}$ & \\
H9 & 0.3531 & 0.5391 & -0.0264 & $0.041^{*}$ & \\
C3 & $0.48414(15)$ & $0.7131(3)$ & $-0.0271(2)$ & $0.0377(4)$ & \\
H10 & 0.5317 & 0.7769 & 0.0368 & $0.045^{*}$ & \\
H11 & 0.4496 & 0.8195 & -0.0780 & $0.045^{*}$ & \\
C4 & $0.44956(15)$ & $0.4235(3)$ & $0.10849(19)$ & $0.0372(4)$ & \\
H12 & 0.3926 & 0.3446 & 0.1458 & $0.045^{*}$ & \\
H13 & 0.4964 & 0.4794 & 0.1759 & $0.045^{*}$ & \\
O4A & $0.2172(5)$ & $0.5036(5)$ & $0.3865(3)$ & $0.0557(14)^{*}$ & $0.563(14)$ \\
H14 & 0.2488 & 0.3733 & 0.3721 & $0.067^{*}$ & \\
H15 & 0.2536 & 0.5828 & 0.3296 & $0.067^{*}$ & \\
O4B & $0.2678(6)$ & $0.5011(6)$ & $0.4032(4)$ & $0.0507(17)^{*}$ & \\
& & & & & \\
& & & & \\
\end{tabular}

Atomic displacement parameters $\left(\AA^{2}\right)$

\begin{tabular}{lllllll}
\hline & $U^{11}$ & $U^{22}$ & $U^{33}$ & $U^{12}$ & $U^{13}$ & $U^{23}$ \\
\hline P1 & $0.0236(2)$ & $0.0260(2)$ & $0.0267(2)$ & $0.00143(15)$ & $0.00188(16)$ & $0.00038(16)$ \\
O1 & $0.0408(7)$ & $0.0355(7)$ & $0.0416(8)$ & $0.0016(6)$ & $-0.0139(6)$ & $-0.0054(6)$ \\
O2 & $0.0250(6)$ & $0.0369(7)$ & $0.0388(7)$ & $-0.0022(5)$ & $0.0023(5)$ & $-0.0088(5)$ \\
O3 & $0.0453(8)$ & $0.0385(8)$ & $0.0431(8)$ & $0.0168(6)$ & $0.0109(6)$ & $-0.0020(6)$ \\
N1 & $0.0276(7)$ & $0.0276(7)$ & $0.0264(7)$ & $-0.0051(5)$ & $0.0022(5)$ & $-0.0039(5)$ \\
C1 & $0.0364(8)$ & $0.0248(8)$ & $0.0339(9)$ & $0.0031(7)$ & $-0.0010(7)$ & $0.0004(7)$ \\
C2 & $0.0282(8)$ & $0.0357(9)$ & $0.0319(9)$ & $0.0025(7)$ & $-0.0033(7)$ & $-0.0021(7)$ \\
N2 & $0.0235(7)$ & $0.0417(9)$ & $0.0365(8)$ & $0.0052(6)$ & $0.0004(6)$ & $-0.0117(7)$ \\
C3 & $0.0351(9)$ & $0.0280(9)$ & $0.0496(11)$ & $0.0007(7)$ & $-0.0025(8)$ & $-0.0020(8)$
\end{tabular}




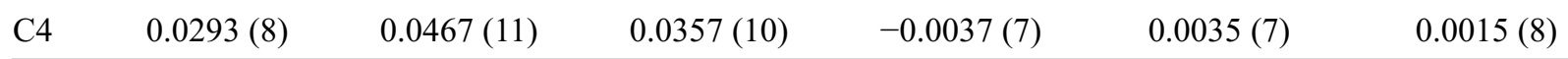

Geometric parameters $(\AA, \stackrel{o}{)}$

\begin{tabular}{|c|c|c|c|}
\hline $\mathrm{P} 1-\mathrm{O} 3$ & $1.5151(13)$ & $\mathrm{N} 2-\mathrm{C} 3$ & $1.479(2)$ \\
\hline $\mathrm{P} 1-\mathrm{O} 2$ & $1.5230(12)$ & $\mathrm{N} 2-\mathrm{H} 8$ & 0.9000 \\
\hline $\mathrm{P} 1-\mathrm{O} 1$ & $1.5234(14)$ & $\mathrm{N} 2-\mathrm{H} 9$ & 0.9000 \\
\hline $\mathrm{P} 1-\mathrm{H} 1$ & 1.3200 & $\mathrm{C} 3-\mathrm{C} 4^{\mathrm{ii}}$ & $1.512(3)$ \\
\hline $\mathrm{N} 1-\mathrm{C} 1$ & $1.488(2)$ & $\mathrm{C} 3-\mathrm{H} 10$ & 0.9700 \\
\hline $\mathrm{N} 1-\mathrm{C} 2$ & $1.492(2)$ & $\mathrm{C} 3-\mathrm{H} 11$ & 0.9700 \\
\hline $\mathrm{N} 1-\mathrm{H} 2$ & 0.9000 & $\mathrm{C} 4-\mathrm{C} 3^{\mathrm{ii}}$ & $1.512(3)$ \\
\hline $\mathrm{N} 1-\mathrm{H} 3$ & 0.9000 & $\mathrm{C} 4-\mathrm{H} 12$ & 0.9700 \\
\hline $\mathrm{C} 1-\mathrm{C} 2^{\mathrm{i}}$ & $1.513(2)$ & $\mathrm{C} 4-\mathrm{H} 13$ & 0.9700 \\
\hline $\mathrm{C} 1-\mathrm{H} 4$ & 0.9700 & $\mathrm{O} 4 \mathrm{~A}-\mathrm{O} 4 \mathrm{~B}$ & $0.638(5)$ \\
\hline $\mathrm{C} 1-\mathrm{H} 5$ & 0.9700 & $\mathrm{O} 4 \mathrm{~A}-\mathrm{H} 14$ & 0.9549 \\
\hline $\mathrm{C} 2-\mathrm{C}^{\mathrm{i}}$ & $1.513(2)$ & $\mathrm{O} 4 \mathrm{~A}-\mathrm{H} 15$ & 0.9296 \\
\hline $\mathrm{C} 2-\mathrm{H} 6$ & 0.9700 & $\mathrm{O} 4 \mathrm{~B}-\mathrm{H} 14$ & 0.9285 \\
\hline $\mathrm{C} 2-\mathrm{H} 7$ & 0.9700 & $\mathrm{O} 4 \mathrm{~B}-\mathrm{H} 15$ & 0.9594 \\
\hline $\mathrm{N} 2-\mathrm{C} 4$ & $1.477(3)$ & & \\
\hline $\mathrm{O} 3-\mathrm{P} 1-\mathrm{O} 2$ & $113.03(8)$ & $\mathrm{C} 4-\mathrm{N} 2-\mathrm{C} 3$ & $111.13(14)$ \\
\hline $\mathrm{O} 3-\mathrm{P} 1-\mathrm{O} 1$ & $112.44(8)$ & $\mathrm{C} 4-\mathrm{N} 2-\mathrm{H} 8$ & 109.4 \\
\hline $\mathrm{O} 2-\mathrm{P} 1-\mathrm{O} 1$ & $111.96(8)$ & $\mathrm{C} 3-\mathrm{N} 2-\mathrm{H} 8$ & 109.4 \\
\hline $\mathrm{O} 3-\mathrm{P} 1-\mathrm{H} 1$ & 106.3 & $\mathrm{C} 4-\mathrm{N} 2-\mathrm{H} 9$ & 109.4 \\
\hline $\mathrm{O} 2-\mathrm{P} 1-\mathrm{H} 1$ & 106.3 & $\mathrm{C} 3-\mathrm{N} 2-\mathrm{H} 9$ & 109.4 \\
\hline $\mathrm{O} 1-\mathrm{P} 1-\mathrm{H} 1$ & 106.3 & $\mathrm{H} 8-\mathrm{N} 2-\mathrm{H} 9$ & 108.0 \\
\hline $\mathrm{C} 1-\mathrm{N} 1-\mathrm{C} 2$ & $111.10(13)$ & $\mathrm{N} 2-\mathrm{C} 3-\mathrm{C}^{4 i}$ & $109.44(15)$ \\
\hline $\mathrm{C} 1-\mathrm{N} 1-\mathrm{H} 2$ & 109.4 & $\mathrm{~N} 2-\mathrm{C} 3-\mathrm{H} 10$ & 109.8 \\
\hline $\mathrm{C} 2-\mathrm{N} 1-\mathrm{H} 2$ & 109.4 & $\mathrm{C} 4 \mathrm{ii}-\mathrm{C} 3-\mathrm{H} 10$ & 109.8 \\
\hline $\mathrm{C} 1-\mathrm{N} 1-\mathrm{H} 3$ & 109.4 & $\mathrm{~N} 2-\mathrm{C} 3-\mathrm{H} 11$ & 109.8 \\
\hline $\mathrm{C} 2-\mathrm{N} 1-\mathrm{H} 3$ & 109.4 & $\mathrm{C} 4 \mathrm{ii}-\mathrm{C} 3-\mathrm{H} 11$ & 109.8 \\
\hline $\mathrm{H} 2-\mathrm{N} 1-\mathrm{H} 3$ & 108.0 & $\mathrm{H} 10-\mathrm{C} 3-\mathrm{H} 11$ & 108.2 \\
\hline $\mathrm{N} 1-\mathrm{C} 1-\mathrm{C} 2^{\mathrm{i}}$ & $110.40(14)$ & $\mathrm{N} 2-\mathrm{C} 4-\mathrm{C} 3^{\mathrm{ii}}$ & $110.48(16)$ \\
\hline $\mathrm{N} 1-\mathrm{C} 1-\mathrm{H} 4$ & 109.6 & $\mathrm{~N} 2-\mathrm{C} 4-\mathrm{H} 12$ & 109.6 \\
\hline $\mathrm{C} 2 \mathrm{i}-\mathrm{C} 1-\mathrm{H} 4$ & 109.6 & $\mathrm{C} 33^{\mathrm{ii}}-\mathrm{C} 4-\mathrm{H} 12$ & 109.6 \\
\hline $\mathrm{N} 1-\mathrm{C} 1-\mathrm{H} 5$ & 109.6 & $\mathrm{~N} 2-\mathrm{C} 4-\mathrm{H} 13$ & 109.6 \\
\hline $\mathrm{C} 2 \mathrm{i}-\mathrm{C} 1-\mathrm{H} 5$ & 109.6 & $\mathrm{C}^{3 \mathrm{ii}}-\mathrm{C} 4-\mathrm{H} 13$ & 109.6 \\
\hline $\mathrm{H} 4-\mathrm{C} 1-\mathrm{H} 5$ & 108.1 & $\mathrm{H} 12-\mathrm{C} 4-\mathrm{H} 13$ & 108.1 \\
\hline $\mathrm{N} 1-\mathrm{C} 2-\mathrm{C}^{\mathrm{i}}$ & $110.45(14)$ & $\mathrm{O} 4 \mathrm{~B}-\mathrm{O} 4 \mathrm{~A}-\mathrm{H} 14$ & 68.0 \\
\hline $\mathrm{N} 1-\mathrm{C} 2-\mathrm{H} 6$ & 109.6 & $\mathrm{O} 4 \mathrm{~B}-\mathrm{O} 4 \mathrm{~A}-\mathrm{H} 15$ & 72.8 \\
\hline $\mathrm{C} 1 \mathrm{i}-\mathrm{C} 2-\mathrm{H} 6$ & 109.6 & $\mathrm{H} 14-\mathrm{O} 4 \mathrm{~A}-\mathrm{H} 15$ & 100.7 \\
\hline $\mathrm{N} 1-\mathrm{C} 2-\mathrm{H} 7$ & 109.6 & $\mathrm{O} 4 \mathrm{~A}-\mathrm{O} 4 \mathrm{~B}-\mathrm{H} 14$ & 72.5 \\
\hline $\mathrm{C} 1-\mathrm{C} 2-\mathrm{H} 7$ & 109.6 & $\mathrm{O} 4 \mathrm{~A}-\mathrm{O} 4 \mathrm{~B}-\mathrm{H} 15$ & 67.8 \\
\hline $\mathrm{H} 6-\mathrm{C} 2-\mathrm{H} 7$ & 108.1 & $\mathrm{H} 14-\mathrm{O} 4 \mathrm{~B}-\mathrm{H} 15$ & 100.5 \\
\hline
\end{tabular}

Symmetry codes: (i) $-x,-y+1,-z$; (ii) $-x+1,-y+1,-z$. 
supporting information

Hydrogen-bond geometry $\left(\AA,{ }^{\circ}\right)$

\begin{tabular}{lllll}
\hline$D-\mathrm{H} \cdots A$ & $D-\mathrm{H}$ & $\mathrm{H} \cdots A$ & $D \cdots A$ & $D-\mathrm{H}^{\cdots} A$ \\
\hline $\mathrm{N} 1-\mathrm{H} 2 \cdots \mathrm{O} 2^{\mathrm{iii}}$ & 0.90 & 1.84 & $2.7147(19)$ & 164 \\
$\mathrm{~N} 1-\mathrm{H} 3 \cdots \mathrm{O} 2^{\text {iv }}$ & 0.90 & 1.81 & $2.7043(19)$ & 172 \\
$\mathrm{~N} 2-\mathrm{H} 8 \cdots 3^{\text {iv }}$ & 0.90 & 1.77 & $2.642(2)$ & 163 \\
$\mathrm{~N} 2-\mathrm{H} 9 \cdots 1^{v}$ & 0.90 & 1.78 & $2.676(2)$ & 171 \\
$\mathrm{O} 4 A-\mathrm{H} 14 \cdots \mathrm{O} 1$ & 0.95 & 1.90 & $2.840(4)$ & 167 \\
$\mathrm{O} 4 A-\mathrm{H} 15 \cdots \mathrm{O} 3^{\text {iv }}$ & 0.93 & 1.90 & $2.811(4)$ & 168 \\
$\mathrm{O} 4 B-\mathrm{H} 14 \cdots \mathrm{O} 1$ & 0.93 & 1.90 & $2.752(4)$ & 151 \\
$\mathrm{O} 4 B-\mathrm{H} 15 \cdots \mathrm{O} 3^{\text {iv }}$ & 0.96 & 1.90 & $2.765(4)$ & 149 \\
$\mathrm{C} 1-\mathrm{H} 5 \cdots \mathrm{O} 4 A^{\mathrm{iii}}$ & 0.97 & 2.38 & $3.300(5)$ & 159
\end{tabular}

Symmetry codes: (iii) $-x, y+1 / 2,-z+1 / 2$; (iv) $x, y+1, z$; (v) $x,-y+1 / 2, z-1 / 2$. 